\title{
Principales fantasías sexuales en jóvenes españoles y diferencias en función de variables sociodemográficas
}

\author{
DAVID RAYA GÜIMIL \\ al128587@uji.es \\ CARLA IBÁÑEZ NAVA \\ al128608@uji.es \\ IVÁN MARTínEZ NAVARRO \\ al311438@uji.es \\ Pedro Salmerón-SÁnchez \\ psalmero@uji.es \\ RAfael BALlester-ARnal \\ rballest@uji.es
}

\section{Resumen}

Introducción: El concepto fantasía sexual define cualquier pensamiento o imagen que provoca excitación en una persona, sea una historia real, una elaboración imaginaria o un pensamiento fugaz. Son frecuentes durante la masturbación o las relaciones sexuales pero también aparecen en situaciones no sexuales. Nuestro objetivo es analizar la prevalencia de las fantasías sexuales más comunes entre los jóvenes, así como estudiar posibles diferencias según distintas variables demográficas. Metodología: Se analizaron las respuestas de 192 hombres y 208 mujeres entre 18 y 26 años $(M=21,8 ; D T=2,2)$, que contestaron de forma online al cuestionario SFQ (Wilson, 1978), en su adaptación española de Sierra y cols. (2006) versionada para este estudio. Resultados: La edad media de inicio de fantasías sexuales es de 13,5 años (hombres: 12,7 y mujeres: 14,$3 ; t=-6,38 ; p<0,05)$. Se hallaron diferencias significativas en el número de fantasías sexuales que presentaban ambos sexos ( $t=-2,062$; $p<0,05$ ), siendo la media mayor en mujeres, y se encontró una media significativamente superior en el grupo de jóvenes con orientación bisexual respecto al de orientación heterosexual $(F=9,607 ; p<0,05)$. Entre las cinco fantasías sexuales más prevalentes en cada sexo, cuatro de ellas coincidían en hombres y mujeres. Conclusiones: Los hombres experimentan fantasías sexuales a una edad más temprana que las mujeres. Existen diferencias significativas en el número de fantasías sexuales en función del género y la orientación sexual, pero no en su contenido.

Palabras clave: fantasías sexuales, edad de inicio, orientación sexual, acto sexual, población joven. 


\section{Abstract}

Introduction: The concept "sexual fantasy" defines any thought or image that triggers excitation in a person. It might be a real story, a drawing imaginary or a fleeting thought. They are common during masturbation or sexual intercourse but also in non-sexual situations. The aim of this study is to analyze the prevalence of the most common sexual fantasies among youth, as well as to study possible differences based on demographic variables. Methodology: The responses of 192 men and 208 women aged between 18 and $26(M=21.8 ; S D=2.2)$, to our particular online version of the Sierra et al. (2006) Spanish adaptation of the SFQ (Wilson, 1978) were analyzed. Results: The mean age of the onset of sexual fantasies is 13.5 years (men: 12.7 and women: 14.3; $t=-6.381 ; p<0.05)$. Significant differences on the number of sexual fantasies based on sex were found $(t=-2.062 ; p<0.05)$, in which women reported a higher mean. $A$ significant higher mean in the bisexual orientation youth group compared to the heterosexual orientation group was also found $(F=9.607 ; p<0.05)$. Among the five most prevalent sexual fantasies in each sex, men and women shared four of them. Conclusions: Men experience sexual fantasies at an earlier age than women. There are significant differences in the amount of sexual fantasies according to sex and sexual orientation, but not about the subject matter.

Keywords: sexual fantasy, age of the onset, sexual orientation, sexual intercourse, youth.

\section{Introducción}

Las fantasías sexuales hacen referencia a cualquier pensamiento o imagen capaz de provocar excitación en la persona. Pueden estar basadas en historias reales o responder a elaboraciones imaginarias, ya sean bizarras o realistas. Surgen en situaciones sexuales, como la masturbación o las relaciones sexuales, aunque también durante situaciones sin connotación sexual (Leitenberg y Henning, 1995).

Los pensamientos y cogniciones pueden incrementar o inhibir la respuesta sexual a cualquier forma de estimulación sensorial y, en ausencia de estimulación física, la fantasía sexual por sí sola es estimulante. Además, las fantasías sexuales pueden dirigir la conducta futura de una persona, así como reflejar experiencias pasadas, sin ser necesariamente personales.

Debido a que las fantasías sexuales son privadas y no dependen de la participación de ninguna otra persona para poder llevarse a cabo, pueden ser más reveladoras que la conducta real, tanto en hombres como en mujeres (Ellis y Symons, 1990). Pueden, incluso, ser la base de ofensas sexuales como el exhibicionismo, la violación y el abuso sexual, según su contenido. En el estudio realizado por Joyal, Cossette y Lapierre (2015) en el que se trabajó sobre la definición de las fantasías sexuales inusuales y raras, se encontró que dos fantasías sexuales fueron insólitas (expresadas por menos del 2,3 \% de los participantes): tener sexo con un niño de una edad menor a 12 años y tener sexo con un animal.

Por otra parte, su ausencia o la culpa generada acerca de tenerlas puede contribuir al desarrollo de disfunciones sexuales. En el DSM-V (American Psychiatric Association, 2013), la presencia de pensamientos sexuales/eróticos o fantasías es considerada un indicador de 
salud sexual, y la ausencia de estos sería uno de los criterios para diagnosticar un deseo sexual hipoactivo en el hombre y un trastorno del interés/excitación sexual en la mujer (Moyano y Sierra, 2014).

Numerosas investigaciones estudian las posibles diferencias de género a la hora de recurrir a fantasías sexuales. En su estudio, Moyano y Sierra (2014) indican que los hombres tienden a experimentar fantasías sexuales con más frecuencia que las mujeres, siendo ellos quienes presentan, además, más tipos diferentes de fantasías sexuales respecto a las mujeres (Wilson y Lang, 1981). También se ha encontrado que dedican más tiempo a pensar en dormir y comer, por lo que se concluiría que los hombres piensan más en sus necesidades básicas que las mujeres (Fisher, Moore y Pittenger, 2012).

Las fantasías sexuales empiezan a darse de forma regular durante la adolescencia temprana, coincidiendo con un aumento en el deseo y la excitación sexual. Gold y Gold (1991) informaron de que la edad media de aparición de fantasías sexuales en los chicos era a los 11,5 años, mientras que la edad media en las chicas era de 12,9. Alguna de las posibles explicaciones pueden ser las diferentes experiencias socializadoras, más que las capacidades psicológicas. Según el mismo estudio, las chicas eran más propensas a basar sus fantasías sexuales en relaciones que tenían o habían tenido, mientras que los hombres se basaban más en estímulos visuales.

Líneas de investigaciones actuales apuntan a la importancia del cerebro, así como de los órganos sexuales, en lo que se refiere al sexo, por lo que surgen las inquietudes acerca de si existen diferencias cerebrales, según género, a la hora de recurrir a las fantasías sexuales.

Por tanto, parece vital comprender las fantasías sexuales en el ámbito de la sexualidad y la salud humana. Sin embargo, su carácter subjetivo y cualitativo, unido a que sus descripciones son, en su mayoría, de carácter excitatorio, hacen que estas no hayan recibido demasiada importancia empírica por el momento.

Esta investigación tiene como objetivo estudiar la prevalencia de las fantasías sexuales más comunes en población jóven española, la edad de inicio a la que los sujetos empiezan a experimentarlas, el número de fantasías sexuales que presentan hombres y mujeres recogidas a través de la adaptación española de Sierra, Ortega y Zubeidat (2006) del Sex Fantasy Questionnaire de Wilson (SFQ; Wilson, 1978), así como dar respuesta a preguntas tales como: ¿existen diferencias de género en el contenido y la frecuencia de las fantasías sexuales?, ¿varían las fantasías sexuales en función de la orientación sexual?, ¿en qué momentos se recurre más a ellas? y ¿qué estímulos provocan excitación sexual?

\section{Método}

\section{Muestra y recogida de datos}

En esta investigación se pretendía conseguir una muestra representativa de la población joven para explorar en ella las fantasías sexuales más prevalentes. Se obtuvo inicialmente una muestra de 1000 sujetos, de los cuales se descartaron aquellos con edades superiores a 26 años. A partir de la muestra original, se seleccionaron de forma aleatoria un total de 192 hombres y 208 mujeres con edades comprendidas entre los 18 y 26 años ( $M=21,8$; DT = 2,2), creándose una nueva muestra con un $n=400$.

La población era mayoritariamente española $(93,8 \%)$, universitaria $(78,3 \%)$, heterosexual $(68,3 \%)$, soltera $(98,0 \%)$, con pareja estable $(50,8 \%)$, con orientación política mayoritariamente progresista $(78,3 \%)$, y de creencia atea $(49,5 \%)$. 
Los datos de la muestra se obtuvieron a partir de un cuestionario en línea publicado en la plataforma virtual de Google Docs. El grupo de investigación preguntaba aleatoriamente a estudiantes del campus de la Universitat Jaume I si deseaban participar en una investigación sobre fantasías sexuales a cargo del departamento de Unisexsida y el Máster en Psicología General Sanitaria de la misma universidad. Si accedían a participar, se les facilitaba el enlace al cuestionario online a través de su correo electrónico. Así mismo, dicho enlace se compartió en diferentes redes sociales. Se estableció una ventana temporal de dos semanas para la recogida de datos.

\section{Medidas e instrumentos}

El instrumento de evaluación utilizado en este estudio ha sido la adaptación española de Sierra y cols. (2006) del Sex Fantasy Questionnaire de Wilson (SFQ; Wilson, 1978). Dicha adaptación surge de la reducción del número de ítems originales a 24 , a partir de un análisis exploratorio factorial. Cada ítem hace referencia a un tipo de fanstasía sexual. En esta investigación se ha trasladado a formato de Google Docs una versión adaptada de Sierra y cols. (2006).

Dentro de cada ítem se preguntó a la persona si había tenido la fantasía sexual en cuestión en algún momento de su vida y, en caso afirmativo, debía establecer el grado de satisfacción que le provocaba, responder si la había llevado a cabo o, en caso contrario, si le gustaría hacerlo.

Además, se incluyó una sección preliminar donde se recogían variables demográficas como sexo, edad, nivel de estudios, orientación política, creencia religiosa, estado civil y situación sentimental actual. Respecto a cuestiones relacionadas con fantasías sexuales, se preguntó la edad a la que el sujeto comenzó a tener fantasías sexuales.

\section{Análisis de datos}

Se realizaron distintos análisis estadísticos mediante el programa estadístico IBM SPSS $^{\circledR}$ statistics 22. Principalmente se realizaron análisis de frecuencias y descriptivos para obtener los datos demográficos de la muestra; diferencias de medias (pruebas $t$ ) para ver si existían diferencias significativas en muestras independientes (hombres y mujeres) en alguna de las variables y análisis de varianza (ANOVA) con objeto de observar si existían diferencias significativas entre grupos de sujetos (por ejemplo, según orientación sexual).

La mayoría de análisis estadísticos se han basado en la frecuencia de respuestas obtenidas y en sus correspondientes porcentajes, dado que la mayoría de ítems del cuestionario representaban variables categoriales.

\section{Resultados}

Se obtuvo una media muestral de 13,5 años. Los hombres afirmaban haber tenido su primera fantasía sexual sobre los 12,7 años, mientras que las mujeres indicaban una edad mayor de inicio, situándola en torno a los 14,3 años. Se hallaron diferencias significativas en la edad de inicio según sexo $(t=-6,381 ; p<0,01)$.

Se analizó la media en el número de fantasías sexuales que presentaban los participantes. Se hallaron diferencias significativas en el número de fantasías sexuales que presentaban hombres $(M=12,8$; $D T=4,26)$ y mujeres $(M=13,7$; $D T=4,33)$, siendo la media mayor en mujeres $(t=-2,062 ; p<0,05)$. 
También se encontraron diferencias significativas según orientación sexual, encontrando diferencias significativas entre los tres grupos $(F=9,607 ; p<0,01)$. Si comparamos entre grupos (véase la tabla 1), la diferencia entre la media de sujetos con conductas heterosexuales y homosexuales no fue significativa pero sí entre sujetos con conductas heterosexuales y bisexuales, siendo la media de estos últimos mayor.

Tabla 1

Media de fantasías sexuales según orientación sexual

\begin{tabular}{lccc}
\hline & $\mathrm{N}$ & Media & $\mathrm{DT}$ \\
\hline Heterosexual & 273 & 12,67 & 4,28 \\
Homosexual & 53 & 13,74 & 3,82 \\
Bisexual & 74 & 15,04 & 4,26 \\
Total & 400 & 13,25 & 4,31 \\
\hline
\end{tabular}

A partir de las respuestas de los participantes, se establecieron los rankings de las fantasías más frecuentes según sexo, junto con el porcentaje de sujetos que afirmaron haber tenido dicha fantasía sexual en algún momento de su vida. Así mismo, se muestra el nivel de excitación media de cada una de las fantasías en una escala de 0 a 10.

\section{Hombres}

A continuación, se muestra el ranking de fantasías más frecuentes entre los hombres (véase la tabla 2).

Tabla 2

Fantasías más frecuentes en hombres

\begin{tabular}{llc}
\hline & & Porcentaje (\%) \\
\hline 1. & Acto sexual con otras personas (i.e., realizar un trío) & 92,19 \\
2. & Practicar un coito con alguien conocido, pero con el que no se han tenido & 97,67 \\
& relaciones sexuales & \\
3. Acto sexual en lugar distinto al dormitorio (p. ej., en la cocina) & 88,02 \\
4. Practicar sexo oral activo (i.e. practicar cunnilingus o una felación) & 87,5 \\
5. Ser masturbado/a hasta el orgasmo por tu pareja & 79,17 \\
\hline
\end{tabular}

Se observó cómo el nivel de excitación (véase la tabla 3) no se asociaba con la posición en el ranking, puesto que la fantasía de «practicar sexo oral activo», situada en la cuarta posición, fue la que mayor excitación media provocaba en los participantes masculinos, mientras que la primera («acto sexual con otras personas»), señalada por un $92,2 \%$ de los participan- 
tes, fue la que menos excitación les producía. Respecto a esta fantasía, podemos observar que el momento en que los participantes afirmaron tenerla difiere según la orientación sexual (véase la tabla 4).

Tabla 3

Media de excitación de las cinco fantasías más frecuentes en hombres

\begin{tabular}{lccccc}
\hline & $1 .^{\circ}$ & $2 .^{\circ}$ & $3 .^{\circ}$ & $4 .^{\circ}$ & $5 .^{\circ}$ \\
\hline Media & 7,20 & 7,59 & 7,31 & 7,87 & 7,63 \\
DT & 2,18 & 2,18 & 2,28 & 2,15 & 2,13 \\
\hline
\end{tabular}

Tabla 4

Momento en el que los hombres suelen tener la fantasía sexual más prevalente

\begin{tabular}{llccc}
\hline & & \multicolumn{3}{c}{ Orientación sexual } \\
& & Heter. & Homo. & Bis. \\
\hline \multirow{2}{*}{$\begin{array}{l}\text { Acto sexual con otras } \\
\text { personas (i.e., realizar un } \\
\text { trío) }\end{array}$} & Durante actividades no sexuales & $50,5 \%$ & $38,9 \%$ & $38,1 \%$ \\
& Durante masturbación & $45,0 \%$ & $58,3 \%$ & $52,4 \%$ \\
& Durante relaciones sexuales & $4,5 \%$ & $2,8 \%$ & $9,5 \%$ \\
\hline
\end{tabular}

\section{Mujeres}

Si tenemos en cuenta el ranking de fantasías más frecuentes en mujeres (véase la tabla 5), se observa una coincidencia entre hombres y mujeres en cuatro de las cinco fantasías sexuales más prevalentes, aunque en distinto orden. Las fantasías no coincidentes fueron «Practicar un coito con una pareja amada» (presente en mujeres y no en hombres) y «Practicar sexo oral activo» (presente en hombres pero no en mujeres).

Tabla 5

Fantasías más frecuentes en mujeres

\begin{tabular}{lc}
\hline & Porcentaje (\%) \\
\hline 1. Acto sexual en lugar distinto al dormitorio (p. ej., en la cocina) & 94,71 \\
2. Acto sexual con otras personas (i.e., realizar un trío) & 86,06 \\
3. Practicar un coito con una pareja amada & 86,06 \\
4. Practicar un coito con alguien conocido, pero con el que no se han tenido & 86,06 \\
relaciones sexuales & 83,17 \\
\hline
\end{tabular}


Al igual que sucede en los hombres, en el caso de las mujeres es también una de las últimas fantasías del ranking la que mayor excitación les provocaba (véase la tabla 6).

Tabla 6

Media de excitación de las cinco fantasías más frecuentes en mujeres

\begin{tabular}{lccccc}
\hline & $1 .^{\circ}$ & $2 .^{\circ}$ & $3 .^{\circ}$ & $4 .^{\circ}$ & $5 .^{\circ}$ \\
\hline Media & 7,34 & 6,24 & 6,43 & 8,04 & 7,42 \\
DT & 2,22 & 2,69 & 2,43 & 2,24 & 2,53 \\
\hline
\end{tabular}

Respecto a la fantasía más prevalente en mujeres, podemos observar que el momento en que las participantes afirmaron tenerla (véase tabla 7) difiriere respecto al caso de los hombres: mujeres con conductas heterosexuales $(47,4 \%)$ y bisexuales $(51,3 \%)$ afirmaron tenerla durante sus relaciones sexuales (a diferencia de los hombres con conductas heterosexuales), mientras que las mujeres con conductas homosexuales (al igual que sus compañeros masculinos) refirieron experimentarla durante la masturbación.

Tabla 7

Momento en el que las mujeres suelen tener la fantasía sexual más prevalente

\begin{tabular}{llllc}
\hline & & \multicolumn{3}{c}{ Orientación sexual } \\
& & Heter. & Homo. & Bis. \\
\hline Acto sexual en lugar & Durante actividades no sexuales & $35,8 \%$ & $30,8 \%$ & $28,2 \%$ \\
distinto al dormitorio & Durante masturbación & $16,8 \%$ & $38,5 \%$ & $20,5 \%$ \\
(p. ej., en la cocina) & Durante relaciones sexuales & $47,4 \%$ & $30,8 \%$ & $51,3 \%$ \\
\hline
\end{tabular}

\section{Discusión y conclusiones}

¿Qué novedades aporta nuestra investigación respecto a la temática de las fantasías sexuales con respecto a estudios anteriores? Por lo que concierne a la edad de inicio de las fantasías sexuales, podemos afirmar que, en la actualidad y en nuestro estudio, los jóvenes españoles comienzan a fantasear a una edad más tardía respecto a los resultados de Gold y Gold (1991), pasando de 11,5 a 12,7 la media de edad en los hombres y de 12,9 a 14,3 años en las mujeres. Por lo tanto, continuarían siendo los hombres quienes comienzan a fantasear primero.

En cuanto a si existen diferencias de género a la hora de fantasear, hemos hallado que son las mujeres las que más realizan esta acción, ya que la diferencia entre las medias obtenidas fue estadísticamente significativa. Esto iría en contra de los resultados obtenidos por Leitenberg y Henning (1995) quienes aseguraban que los hombres fantaseaban más que las mujeres. 
Al comparar las medias obtenidas sobre las fantasías sexuales más predominantes en la muestra poblacional, según género, de entre las que hace referencia el cuestionario utilizado se encontró que no existen diferencias significativas entre hombres y mujeres; sin embargo, al comparar los mismos índices según la variable orientación sexual, sí se encontraron diferencias significativas entre sujetos con conductas bisexuales y heterosexuales, siendo los sujetos con conductas bisexuales los que más fantasías presentaban.

De igual modo, se estableció a partir de las 24 fantasías recogidas en el SFQ, el ranking de las cinco más prevalentes en la población según género, encontrando que las fantasías «Acto sexual con otras personas», "Practicar un coito con alguien conocido, pero con el que no se han tenido relaciones sexuales», "Acto sexual en lugar distinto al dormitorio (p. ej., en la cocina)» y «Ser masturbado/a hasta el orgasmo por tu pareja» fueron comunes en hombres y mujeres, aunque no en el mismo orden, mientras que «Practicar sexo oral activo (p. ej., practicar cunnilingus o una felación)» fue también favorita entre el sexo masculino y «Practicar un coito con una pareja amada» entre el sexo femenino. Esto haría referencia a la aportación de diversas investigaciones que respaldan la idea de que las fantasías sexuales de las mujeres contienen más elementos como el afecto, la emoción y el argumento (Ellis y Simons, 1990; Follingstad y Krimbell, 1986; Gil, 1990; Hardin y Gold, 1988; Kelley, 1984).

En su revisión de varios estudios anteriores, Leitenberg y Henning (1995) encontraron que un $85,9 \%$ de hombres y un $68,8 \%$ de mujeres fantaseaba durante la masturbación. En nuestra investigación, se han encontrado evidencias de que la fantasía más prevalente en mujeres, «Acto sexual en lugar distinto al dormitorio (p. ej., en la cocina)», se da en mayor medida durante relaciones sexuales en mujeres con conducta heterosexual y bisexual y durante masturbación en mujeres con conducta homosexual. En cuanto al sexo masculino, la fantasía sexual más prevalente, "Acto sexual con otras personas», aparece con más frecuencia durante actividades no sexuales en hombres con conducta heterosexual y en mayor medida durante la masturbación en hombres con conductas homosexuales y bisexuales.

A pesar de que las fantasías sexuales sigan considerándose en gran medida un tema tabú en nuestra sociedad actual, lo cierto es que un gran porcentaje de hombres y mujeres experimentan estos pensamientos con frecuencia. En contra de lo que muestran estudios anteriores, podemos decir que las mujeres fantasean más que los hombres, aunque estas fantasías se sitúan en líneas muy similares, coincidiendo en aspectos como la temática, el contexto y el lugar de las mismas. También hemos podido encontrar diferencias por lo que respecta a la frecuencia de las fantasías en función de la orientación del deseo sexual, así como en el momento en que se recurre a ellas.

\section{Futuras investigaciones}

De cara a futuras investigaciones, planteamos una serie de medidas para mejorar nuestro estudio. En primer lugar, creemos conveniente especificar los contenidos de algunos ítems tras la adaptación española. Puede ser que algún ítem resulte ambiguo y esto produzca errores de comprensión, que luego se traducen en respuestas erróneas.

Por otra parte, sería interesante analizar el momento de aparición del resto de fantasías sexuales más prevalentes. De esta forma podremos aportar una visión más amplia de las fantasías sexuales y comprobar si hay diferencias entre diversas fantasías.

También sería conveniente equilibrar nuestra muestra según la orientación del deseo sexual. Si bien es sabido que hay una menor frecuencia de hombres y mujeres con conductas homosexuales y bisexuales entre la población, debemos intentar igualar la muestra, ya que 
esto nos permitirá indagar más sobre las fantasías sexuales de las diferentes orientaciones sexuales y poder realizar análisis estadísticos con más validez.

Profundizar sobre qué factor provoca que las fantasías más excitantes no sean las más prevalentes es otra cuestión a estudiar en futuras investigaciones. Este dato nos ha parecido muy relevante, ya que la mayoría de sujetos contestaron que no habían llevado a cabo la fantasía sexual, lo que podría ir en relación con la propia excitación en el plano cognitivo y no tanto con el hecho de llevarla a cabo.

\section{Referencias bibliográficas}

American Psychiatric Association (2013). Manual diagnóstico y estadístico de los trastornos mentales (DSM-5). Barcelona: Masson.

Ellis, B. J. y Symons, D. (1990). Sex differences in sexual fantasy: An evolutionary psychological approach. Journal of Sex Research, 27, 527-555.

Fisher, T. D., Moore, Z, T. y Pittenger, M.J. (2012). Sex on the Brain?: An examination of frequency of sexual cognitions as a function of gender, erotophilia, and social desirability. Journal of Sex Research, 49, 69-77.

Follingstad, D. R. y Kimbrell, C. D. (1986). Sex fantasies revisited: An expansion and further clarification of variables affecting sex fantasy production. Archives of Sexual Behavior, $15,475-486$.

Gil, V. E. (1990). Sexual fantasy experiences and guilt among conservative Christians: An exploratory study. Journal of Sex Research, 27, 629-630.

Gold, S. R. y Gold, R. G. (1991). Gender differences in first sexual fantasies. Journal of Sex Education and Therapy, 17, 207-216.

Hardin, K. y Gold, S. (1988). Relationship of sex, sex guilt, and experience to written sexual fantasies. Imagination, Cognition, and Personality, 8, 155-163.

IBM Corp. Released (2013). IBM SPSS Statistics for Windows, Version 22.0. Armonk, NY: IBM Corp.

Joyal, C. C., Cossette, A. y Lapierre, V. (2015). What Exactly Is an Unusual Sexual Fantasy? The Journal of Sexual Medicine, 12, 328-340.

Kelley, K. (1984). Sexual fantasy and attitudes as functions of sex of subject and content of erotica. Imagination, Cognition, and Personality, 4, 339-347.

Leitenberg, H. y Henning, K. (1995). Sexual Fantasy. Psychological Bulletin, 3, 469-496.

Moyano, N. y Sierra, J. C. (2014). Fantasías y pensamientos sexuales: Revisión conceptual y relación con la salud sexual. Revista Puertorriqueña de Psicología, 25, 376-393.

Sierra, J. C., Ortega, V. y Zubeidat, I. (2006). Confirmatory Factor Analysis of a Spanish Version of the Sex Fantasy Questionnaire: Assessing Gender Differences. Journal of Sex y Marital Therapy, 32, 137-159.

Wilson, G. D. (1978). The Secrets of Sexual Fantasy. London: Dent.

Wilson, G. D. (1987). Male-female differences in sexual activity, enjoyment and fantasies. Personality and Individual Differences, 8, 125-127.

Wilson, G. D. y Lang, R. J. (1981). Sex differences in sexual fantasy patterns. Personality and Individual Differences, 2, 343-346. 